\title{
Nonalcoholic fatty liver disease: molecular pathways and therapeutic strategies
}

\author{
Yue Ye Huang ${ }^{1}$, Aaron M Gusdon ${ }^{3}$ and Shen $\mathrm{Qu}^{1,2^{*}}$
}

\begin{abstract}
Along with rising numbers of patients with metabolic syndrome, the prevalence of nonalcoholic fatty liver disease (NAFLD) has increased in proportion with the obesity epidemic. While there are no established treatments for NAFLD, current research is targeting new molecular mechanisms that underlie NAFLD and associated metabolic disorders. This review discusses some of these emerging molecular mechanisms and their therapeutic implications for the treatment of NAFLD. The basic research that has identified potential molecular targets for pharmacotherapy will be outlined.
\end{abstract}

Keywords: Nonalcoholic fatty liver disease, Molecular mechanisms

The prevalence of obesity is increasing worldwide. While there are no established treatments for NAFLD, however current research is targeting new molecular mechanisms that underlie NAFLD and associated metabolic disorders.

\section{Nuclear transcription factors: PXR and FXR}

Studies in mice have shown that the Pregnane X Receptor (PXR), Farnesoid X Receptor (FXR), and Forkhead $\mathrm{O} 1$ are implicated in hepatic steatosis and in lipid and glucose metabolism [1]. It is accepted that nuclear transcription factors are major regulators of the metabolism of lipid and glucose but the specific pathways and interrelationships are still being investigated. PXR was initially found to activate transcription of steroid-inducible genes by forming a heterodimer with RXR and binding to PXR response elements in response to a number of different steroid precursors and metabolites as well as other both natural and synthetic compounds [2]. Many studies have demonstrated that PXR plays an important role in the clearance and elimination of toxic products of metabolism [3], however it has become clear that PXR also plays an important role in lipid metabolism as it was shown that PXR could increase fatty acid uptake

\footnotetext{
* Correspondence: qushencn@hotmail.com

'Department of Endocrinology and Metabolism, Shanghai 10th People's Hospital, School of Medicine, Tongji University, Shanghai 200072, China ${ }^{2}$ Department of Endocrinology and Metabolism, Nanjing Medical University, Nanjing 210029, Jiangsu Province, China

Full list of author information is available at the end of the article
}

by directly increasing the expression of the membrane bound fatty acid transporter (FAT, CD36) [4] PXR transgenic mice demonstrated hepatic steatosis [1]. Further studies have shown that PXR exerts its effect on lipid metabolism through several distinct mechanisms. In addition to its first known role in increasing fatty acid uptake into hepatocytes, PXR is also able to increase lipogenesis while also down regulating fatty acid beta oxidation. PXR's ability to increase lipogenesis is due to enhanced transcription of stearoyl-CoA desaturase, fatty acid elongase, fatty acid synthase, and ATP citrate lyase [5]. These effects are largely due to the interaction of PXR with S14, which is response to various nutrient and hormonal signals [5]. The ability of PXR to downregulate fatty acid beta oxidation is largely due to its ability to impair signaling through the forkhead transcription factor Foxa2. Foxa2 induces transcription of genes necessary for fatty acid oxidation and ketogenesis and it is rendered inactive in the cytoplasm by insulin [6]. Foxa2 mediates the upregulation of carnitine palmitoyltransferase (Cpt1a, involved in beta-oxidation) and 3-hydroxy-3methylglutarate-CoA synthase 2 (Hmgcs2, involved in ketogenesis), and PXR is able to directly bind Foxa2 and impair its ability to upregulate these genes [7]. Additionally, PXR has been shown to interact with the forkhead box-containing protein $\mathrm{O}$ subfamily (FOXO1). FOXO1 regulates the expression of several enzymes involved in gluconeogenesis and FOXO1 mRNA levels have been shown to correlate with the severity of NASH [8]. FOXO1 
can act as a coactivator of PXR mediated transcription, however, their interaction is complex, and under the presence of the appropriate activator, PXR can act as a corepressor of FOXO1 mediated transcription [9].

Farnesoid X receptor (FXR) was originally described as a farnesol-activated receptor and has long been known for its function as a bile acid sensor in enterohepatic tissues [10]. FXR has emerged in recent years as a master regulator of lipid and glucose homeostasis in the liver and of inflammatory processes at hepatic and extrahepatic sites, and a number of synthetic FXR agonists are being tested for the treatment of different hepatic and metabolic disorders $[11,12]$ given the ability of FXR to antagonize inflammatory and fibrogenic processes. Two FXR genes have been identified, FXR $\alpha$ and FXR $\beta$. FXR $\alpha$ is expressed mainly in the liver, intestines, kidney, and adrenal glands, and at much lower levels in adipose tissues. FXR $\beta$ is a lanosterol sensor that encodes a functional protein in rodents but not in humans [13]. FXR is an obligate partner of the 9-cisretinoic acid receptor RXR (retinoid X receptor). The relevance of FXR to hepatic physiology and disease became apparent from the observation that FXR-knockout mice on a high-fat diet exhibit hyperlipidemia and massive hepatic steatosis, as well as necroinflammation and fibrogenesis $[14,15]$. Importantly, a high fat diet led to macrosteatosis without inflammation in the livers of LDL receptor knockout mice whereas the livers of mice double-knockout (LDLR-/- and FXR-/-) mice showed necroinflammation and increased hepatic levels of tumor necrosis factor- $\alpha$, intercellular adhesion molecule-1, transforming growth factor (TGF) $-\beta$, procollagen $1 \alpha 1$, and collagen, suggesting FXR may prevent progression of simple steatosis to NASH. FXR induces expression of genes that promote triglyceride clearance and mitochondrial fatty acid $\beta$-oxidation, as well as suppression of lipogenic gene transcription [16]. FXR also upregulates the genes encoding apolipoprotein C-II (apoC-II) and very-low-density lipoprotein receptor, therefore enhancing triglyceride-rich lipoprotein clearance, while apoA-I gene expression is suppressed [17]. Synthetic FXR agonists decrease gluconeogenesis in mice fed a highfat diet by reducing the expression and activity of PEPCK, G6Pase, and fructose-1,6-biphosphatase, and by enhancing insulin signaling through insulin receptor substrate-1 (IRS-1) phosphorylation $[18,19]$. FXR also appears to antagonize hepatic inflammatory and fibrogenetic processes. Another hepatic protective mechanism of FXR activation has been shown to be maintenance of gut integrity against gut-derived endotoxins through induction of antibacterial factors such as angiogenin, iNOS, and interleukin (IL)-18 [20].

\section{PPARs, RXR and FGF2 1}

Three distinct members of the peroxisome proliferator activated receptor (PPAR) sub-family each encoded by a distinct gene have been identified and well characterized. PPAR $\alpha$ (NR1C1) is highly expressed in liver, kidney, and muscle. PPARy (NR1C3) is enriched in adipose tissue and PPAR $\beta / \delta$ (NR1C2; referred to as PPAR $\delta$ in this review) appears to be ubiquitously expressed. All three PPARs bind to DNA as heterodimers with retinoid $\mathrm{X}$ receptors (RXR; NR2B sub-group) and bind preferentially to direct repeats of the nuclear receptor half site AGGTCA separated by 1 nucleotide (DR1). Each sub-type appears to have unique functions and PPAR $\alpha$ and PPAR $\gamma$ are the targets of the fibrate and thiazolidinedione (TZD) classes of drugs respectively.

Studies in vitro and in vivo have demonstrated that PPAR $\alpha$ directly regulates a network of genes encoding the proteins required for the uptake of fatty acids, enzymes required of the oxidation of fatty acids, and enzymes required for ketogenesis by binding to control regions in the promoter of these genes. Thus, activation of PPAR $\alpha$ promotes the utilization of fat as an energy source. Mice lacking PPAR $\alpha$ accumulate triglycerides in the liver and become hypoglycemic during fasting or starvation. Recent studies indicate that fibroblast growth factor 21 (FGF21) functions as an endocrine hormone that mediates many of the effects of PPAR $\alpha$. The gene encoding FGF21is directly induced by PPAR $\alpha$ in response to fasting via a binding site in the promoter. FGF21 in turn stimulates lipolysis in adipose tissue and ketogenesis in the liver. In vitro studies have demonstrated that fatty acids can bind to PPAR $\alpha$ and function as ligands. More recently, affinity purification of PPAR $\alpha$ from mouse livers followed by mass spectrometery identified 1-palmityol-2-oleoyl-sn-glycerol-3-phosphocholine (16:0/18:1-GPC) as a selective PPAR $\alpha$ agonist [21]. Interestingly, the production of 16:0/18:1-GPC requires the activity of fatty acid synthase (FAS) suggesting that PPAR $\alpha$-dependent fat oxidation serves as a regulatory mechanism to ensure that fatty acids levels stay within an optimum range. Taken together PPAR $\alpha$ appears to function as a sensor of the fed/starved state.

PPAR $\gamma$ is a master transcriptional regulator of adipogenesis and plays an important role in the process of lipid storage [22]. Thus PPAR $\alpha$ and PPAR $\gamma$ have opposing functions in the regulation of fat metabolism; PPAR $\alpha$ promotes utilization while activation of PPAR $\gamma$ promotes storage. Indeed, it has been suggested that PPAR $\gamma$ 's role in the activation of lipogenic genes may contribute to the development of steatosis, as increased PPAR $\gamma$ expression has been found in steatotic livers. Nevertheless, several studies have shown that overexpression of PPAR $\gamma$ can prevent the progression of hepatic steatosis in mouse models and treatment with the PPARy agonist rosiglitazone has been shown to have similar effects. The protective effects of PPAR could be due to increased insulin sensitivity in adipose tissue and skeletal muscle leading to decreased 
FFA deposition in the liver. Adiponectin has also been shown to be increased by PPAR $\gamma$ which also contributes to insulin sensitivity as well as up-regulating PPAR $\alpha$ expression, which leads to further hepatic fatty acid oxidation. Furthermore, PPAR $\gamma$ expression has been shown to have anti-inflammatory and anti-fibrotic effects in stellate cells, macrophages, and epithelial cells.

A number of naturally occurring fatty acids and prostanoids have been shown to act as PPAR $\gamma$ agonists, however, perhaps most importantly was the identification that the TZD class of insulin sensitizing drugs including rosiglitazone (Avandia) and pioglitazone (Actos) are PPAR $\gamma$ agonists. Fatty acid accumulation in insulin sensitive tissues such as liver and skeletal muscle has been shown to promote insulin resistance. Activation of PPARy in adipose has been proposed to increase the number of adipocytes and promote the relocalization and storage of fat in adipose tissue, protecting peripheral tissues from lipotoxicity. Consistent with this idea is the observation that selective knockout of PPARY in adipose tissue eliminates the therapeutic activity of TZDs in mice and that a common side effect of TZD in treatment in humans is weight gain due to an increase in adipose mass. The TZDs have proven to be effective drugs for improving insulin sensitivity and treating type II diabetes. Nevertheless, they are not without problems. Troglitazone, the first TZD to be used in the clinic, was taken off the market because of cases of druginduced liver damage. Additionally recent meta-analyses have indicated that treatment with rosiglitazone is associated with increased risk of myocardial infarction and deaths due to cardiovascular events [23]. Pioglitazone treatment was also shown to be associated with an increase in heart failure although a significantly lower risk of myocardial infarction and death was observed in this patient population. Finally, muraglitazar an investigational drug that is a dual agonist of PPAR $\alpha$ and PPAR $\gamma$ was found to be associated with an increase in major cardiovascular events and increased incidence of death. The increases in cardiovascular events and mortality seen with these drugs are relatively small. Nevertheless, the wide scale use of PPAR $\gamma$ agonists in the type II diabetic population has raised serious concerns about the safety of these drugs for long term therapy. The molecular basis underlying the increase in cardiovascular events is not clear. Regulation of the epithelial sodium channel $(\mathrm{ENaC \gamma})$ in the kidney by PPAR $\gamma$ has been suggested as potential mechanism responsible for TZD-dependent edema but it remains to be seen if inhibiting this channel will decrease the cardiovascular events associated with TZD treatment. As the peroxisome proliferator-activated receptors (PPARs) represent major regulators of lipid metabolism in the liver, a few studies have tested the hypothesis that genetic variants in these hormone receptors may influence susceptibility to NAFLD, but the results have been controversial [24-26]. As PPARs are also the target of several drugs under evaluation for the treatment of NAFLD, this evidence may lay the basis to design pharmacogenetic studies to assess the role of PPAR SNPs in predicting the response to drugs targeting these nuclear receptors.

\section{Pex11a}

Peroxisomes are organelles which have important roles in cellular metabolism including very-long chain fatty acid $\beta$-oxidation, cholesterol synthesis, and hydrogen peroxide metabolism [27-29]. Peroxisome proliferation is dependent on Pex11, which has been shown to promote peroxoisome elongation in various model systems [30-34]. Three subtypes of Pex11 have been identified ( $\alpha, \beta$, and $\gamma$ ), with Pex11 $\alpha$ and $\gamma$ being primary expressed in the liver and Pex11 $\beta$ being more broadly expressed [34,35]. Weng et al. [36] recently demonstrated that mice with Pex11 $\alpha$ knocked out had increased body weight and accumulated fat in their livers. Pex $11 \alpha$ knockout mice exhibited elevated levels of free fatty acids without significant effects on glucose or beta-hydroxybutyrate. While loss of Pex $11 \alpha$ impaired peroxisomal elongation, it also decreased the expression of genes important for peroxisomal fatty acid oxidation. Therefore, targeting peroxisomal function may represent a new therapeutic target in the treatment of NAFLD.

\section{P53}

P53 is well known to be a key regulatory of cellular metabolism and has been shown to regulate the balance between oxidative and glycolytic metabolism in cancer cells [37]. Recently, p53 has also been implicated in NAFLD largely through its role in regulating fatty acid oxidation. Similar to its role in alcoholic fatty liver disease, p53 has been shown to be upregulated in the livers of mice with NAFLD [38] and has been linked to hepatocytes apoptosis in NAFLD [39]. To test the viability of p53 as a therapeutic target in NAFLD, Derdak et al. looked at the effect of a p53 inhibitor, pifithrinap-nitro (PFT) on various parameters of NAFLD in a murine model [40]. In HFD fed C57BL/6 mice, PFT treatment was found to reduce hepatic steatosis, apoptosis, and oxidative stress. These effects are believed to be secondary to a reduction in the upregulation of miRNA34a and a shift toward high activity of the SIRT1/AMPK/ACC pathway. SIRT1 increases the expression of AMPK which blocks ACC allowing CPT1 to be activity due to reduced levels of the inhibitory malonyl-CoA. In another arm of the pathway, SIRT1 is also believed to upregulate PGC1 $\alpha$ / PPAR $\alpha$, which upregulates MLYCD subsequently decreasing MLYCD levels, decreasing Malonyl-CoA synthesis and relieving the repression of CPT1. Another recent study confirmed miR-34a upregulation and involvement of SIRT1 and p53 in liver steatosis and found that treatment 
with ursodeoxycholic acid reversed p53 upregulation and restored SIRT1 expression [41]. Taken together, these results support the role of p53 in regulating metabolism and suggest that it may be a target for treatment of NAFLD.

\section{Liver $\mathrm{X}$ receptor}

The Liver X receptor (LXR) sub-group of the nuclear receptor superfamily is comprised of two sub-types, LXR $\alpha$ and LXR $\beta$ that are encoded by separate genes. LXR $\alpha$ was originally cloned from a liver cDNA library and found to be highly expressed in the liver, kidney, and intestine. In contrast, LXR $\beta$ is more ubiquitously expressed. LXRs are important regulators of cholesterol and lipid metabolism, and their activation has been shown to inhibit cardiovascular disease and reduce atherosclerosis in animal models. More recent studies using knockout mice deficient in the synthsis of oxysterols have provided strong support that these cholesterol derivatives function as LXR ligands in vivo [42]. The identification of hydroxycholesterols as natural LXR ligands prompted studies to identify the genetic networks regulating cholesterol metabolism which are controlled by LXR. An increase in hepatic cholesterol levels is predicted to lead to an elevation in the concentration of cholesterol-derived LXR ligands resulting in the catabolism of cholesterol to bile acid and the excretion of cholesterol out of the liver. Indeed, when challenged with a diet rich in cholesterol Lxro-/- mice accumulate massive amounts of cholesterol in the liver. In mice, pharmacological activation of LXR has been shown to result in net movement of cholesterol out of the body, a result consistent with the gene expression and genetic knockout studies. In concert with the elimination of hepatic cholesterol, activation of LXR also decreases cholesterol uptake by inducing expression of the Inducible Degrader of the LDL receptor (Idol) [43]. Over expression of Idol in cell culture models significantly enhances the ubiquitination and proteosome-dependent degradation of the LDL receptor. Additionally, Idol is expressed in macrophages where it may also affect cholesterol uptake.

Along with effects on cholesterol metabolism activation of LXR agonists also increases expression of genes involved in fatty acid metabolism including the master transcriptional regulator of fatty acid synthesis, sterol response element binding protein 1c (SREBP1c) [1]. Several of the genes encoding the enzymes involved in fatty acid metabolism including fatty acid synthase (FAS) and stearoyl CoA desaturase 1 (SCD-1) are directly or indirectly regulated by LXR. The up-regulation of fatty acid synthesis in coordination with reverse cholesterol transport is most likely to provide lipids for the transport and storage of cholesterol. Small molecule agonists of LXR activity are therefore of great therapeutic interest. Nevertheless, the elevations in plasma cholesterol and triglyceride levels seen in LXR agonist-treated animals have severely limited the potential transition of the first generation LXR agonists into the clinic. However, the finding that such agonists also promote hepatic lipogenesis has led to the idea that hepatic LXR activity may be undesirable from a therapeutic perspective. Liver-specific deletion of LXR eliminated the detrimental effect of increased plasma triglycerides, while the beneficial effect of increased plasma HDL was unaltered.

There are numerous studies that have reported LXR plays a major role in the regulation of macrophage reverse cholesterol transportation (RCT) and that will contribute to the lipid accumulation in the liver and development of atherosclerosis. The Liver X Receptors (LXRs) $\alpha$ and $\beta$ and the Peroxisome Proliferator-Activated Receptor $\alpha$ (PPAR $\alpha)$ are transcription factors that belong to class II nuclear receptors. They drive the expression of genes involved in hepatic lipid homeostasis and therefore are important targets for the prevention and treatment of nonalcoholic fatty liver disease (NAFLD). LXRs and PPAR $\alpha$ are regulated by endogenous ligands, oxysterols and fatty acid derived molecules. In the liver, pharmacological activation of LXRs leads to the over-expression of genes involved in de novo lipogenesis, while activation of PPAR $\alpha$ is critical for fatty acid catabolism in nutrient deprivation. While these two nuclear receptors appear to have opposing effects, recent studies have highlighted that PPAR $\alpha$ also influences the expression of genes involved in fatty acids synthesis. T0901317 and fenofibrate induce expression of genes involved inLXR-dependent and PPAR $\alpha$-dependent lipogenic responses. Deficiency of PPAR $\alpha$ does not influence the effects of T0901317 on lipogenic genes expression. However, PPAR $\alpha$ deficiency prevents the up-regulation of genes involved in $\omega$-hydroxylation that are induced by T0901317. In addition, over-expression of lipogenic genes in response to fenofibrate is decreased in LXR knockout mice as well as the expression of PPAR $\alpha$ target genes involved in fatty acid oxidation. This supports the existence of an interaction between these nuclear receptors and suggests LXR and PPAR $\alpha$ may have overlapping control over the expression of hepatic genes involved in lipid metabolism.

\section{Thyroid hormone receptor}

The antiobesity and low-density lipoprotein (LDL)-cholesterol-lowering effects of thyroid hormones have been known for years, but their pharmacological use has been limited by their untoward effects on bone and heart and skeletal muscle. Recent research has developed compounds that lack these adverse effects while harnessing the beneficial effects of thyroid hormone. The development of these drugs, termed thyromimetics, was made possible by the existence of distinct thyroid hormone receptor (TR) subtypes with different tissue-specific distributions [44]. The two subtypes of TRs, TR $\alpha$ and TR $\beta$, are predominantly localized in the heart and liver, respectively. Selective TR $\beta 1$ 
agonists have been synthesized and evaluated in preclinical trials and in a small clinical trial, demonstrating their lipidlowering properties and safety [45]. The liver specificity of these drugs is achieved through the selective hepatic distribution of phosphonic acid: which is phosphonatecontaining TR $\beta 1$ agonist that is taken up predominantly by the liver with a high first pass extraction and converted into the active form in hepatic microsomes by cytochrome P450 3A. Recently, the main compound of this type, MB07811, has been evaluated in different animal models of hepatic steatosis, including ob/ob mice, Zucker diabetic rats, and mice with diet-induced obesity [46]. A marked reduction was observed in plasma cholesterol and triglyceride levels. Hepatic selectivity of MB07811 was demonstrated by showing that this agent did not affect circulating FFA levels, epidydimal fat pad and heart mass, or pituitary thyroid-stimulating hormone mRNA. A decrease in hepatic lipogenesis, as suggested by a reduction in SREBP-1c mRNA, and in hepatic lipase activity, as suggested by reduced levels of apolipoprotein C3 (apo C3, an inhibitor of lipoprotein lipase), might also contribute to the antisteatotic effects of this agent. GC-1, another TR $\beta 1$ selective activator, ameliorated not only steatosis but also markers of hepatic lipoperoxidation and liver injury, suggesting these agents have the potential to reverse not only fat infiltration but also the progressive features of steatohepatitis. How TR mimetics can ameliorate hepatocyte oxidative stress and necroinflammation warrants further investigation. These futile cycles reduce oxidative stress in two ways: they increase ATP cycling, which enhances oxidative phosphorylation and decreases the formation of reactive oxygen species (ROS); and they supply the NADPH necessary for the regeneration of reduced glutathione (GSH) and other reducing substances, thus facilitating the scavenging of ROS [47]. Collectively, the results of the studies show promise for the treatment of NAFLD and associated metabolic disorders, but efficacy and safety of this class of agents in humans need further evaluation.

\section{AMP-activated protein kinanse (AMPK)}

As discussed in previous sections, signaling through AMP can have a beneficial effect on hepatic steatosis. AMPK has emerged as a central regulator of cellular metabolism. AMPK consists of heteromeric complexes with a catalytic $\alpha$ subunit and regulatory $\beta$ and $\gamma$ subunits. AMPK is able to sense the energy status of the cell in a variety of different ways. The $\gamma$ subunits are able to bind adenine nucleotides, with ADP and AMP leading to activation of AMPK though allosteric activation and phosphorylation [48]. AMPK is able to regulate metabolism through its effects on glucose homeostasis, lipid metabolism, protein synthesis, and oxidative metabolism. AMPK exerts its control over glucose metabolism at a number of different steps in glucose utilization. AMPK promotes the upregulation of the proteins needed for glucose uptake into cells [49], phosphorylates 6-phosphofructo-2-kinase (PFK-2) thereby upregulating glycolysis [50], inhibiting gluconeogenesis though multiple mechanisms [51], and phosphorylating and inhibiting glycogen synthase and subsequently reducing the amount of glycogen synthesized [52]. AMPK is able to control lipid metabolism by both decreasing lipogenesis and stimulating mitochondrial fatty acid oxidation. The former task is accomplished by phosphorylating 3hydroxy-3-methylglutaryl-coenzyme A reducase (HMG$\mathrm{CoA}$ ) and acetyl CoA carboxylase 1 (ACC1). Inactivation of HMG-CoA reducase blocks the conversion of HMGCoA to mevalonate and inactivation of ACC1 results in decreased ability to synthesize fatty acids. AMPK's ability to promote fatty acid oxidation is attributed to decreased synthesis of malonyl CoA due to phosphorylation of ACC2 which relieves the inhibition of carnitine O-palmitoyltransferase 1 (CPT1). Recently, AMPK has also been shown to phosphorylate the transcriptional co-activator transducer of regulated CREB activity 2 (TORC2), excluding it from the nucleus and decreasing transcription of the gluconeogenic genes PEPCK and glucose-6-phosphatase [53]. Importantly, AMPK has also been shown to decrease transcription of lipogenic genes thought its inhibition of sterol regulatory element-binding protein-1c (SREBP1c) [54]. Additionally, AMPK has been shown to upregulate oxidative phosphorylation through its ability to positively regulate PGC- $1 \alpha$ through phosphorylation, which subsequently increases mitochondrial biogenesis [55].

Given its ability to sense and regulate the metabolic state of the cell, modulation of AMPK has become an important therapeutic target in NAFLD. Exercise has well known beneficial effects in the metabolic syndrome as well as in NAFLD and some of these effects may be due to its modulation of the AMPK signaling pathway as expression of genes induced by exercise requires AMPK activation [56,57]. Various compounds already in use for the treatment of type 2 diabetes have been shown to have effects on the AMPK pathway. The beneficial effects of thiazolidinediones on hepatic steatosis were discussed above with activation of PPAR $\alpha$ as a driving mechanism. However, the thiazolidinediones have also been shown to increase the AMPK signaling pathway with improvement in both hepatic steatosis and fibrosis [58]. Additionally, biguanides such as metformin have been shown to induce AMPK activity in hepatocytes subsequently increasing fatty acid oxidation, decreasing lipogenesis, and decreasing hepatic glucose production [59]. Treatment with metformin has been shown to reverse liver steatosis and its lipid lower effects were shown to be dependent on AMPK activation [60]. The mechanism by which AMPK is induced by both of these classes of drugs likely involves alteration of mitochondrial function. Thiazolidinediones bind to and 
impair assembly of complex I [61] as well as impairing mitochdrial pyruvate transport [62] and metformin has been shown to inhibit mitochondrial complex I thus decreasing the ATP to ADP/AMP ratio and inducing AMPK.

Polyphenols such as resveratrol [63] and epigallocatechin gallate [64] have been demonstrated to activate AMPK. One suggested mechanism has been the ability of these compounds to activate sirtuin 1 (SIRT1), which acts as a metabolic regulatory through its ability to sense $\mathrm{NAD}+$ levels and also is an upstream regulator of AMPK [65]. Epigallocatechin gallate's may also increase reactive oxygen species production, and subsequently activate CaMKK which can phosphorylate AMPK [66]. However, these compounds also likely act through their effects on adenine nucleotide levels as resveratrol has been shown to inhibit mitochondrial complex V [67] and epigallacatechin gallate has been shown to inhibit mitochondrial complexes I, II, and V [68].

5-aminoimidazole-4-carboxamide-1-b-d-ribofuranoside (AICAR) has been extensively demonstrated to activate hepatic AMPK activity. Treatment with AICAR has been shown to decease hepatic lipid accumulation through AMPK activation [69] and also decrease hepatic glucose production largely through TORC2 inactivation as well as increasing skeletal muscle glucose uptake [70]. While AICAR's effects on AMPK is due to allosteric activation by its metabolite 5 -aminoimidazole-4-carboxamide ribotide (ZMP) which is structurally similar to 5'-AMP, it was originally believed that AICAR treatment did not affect endogenous adenine nucleotide levels. However, recent studies have shown that AICAR treatment results in ATP depletion [71] and can impair mitochondrial function [72], potentially limiting its therapeutic use.

More recently, other activators of AMPK have been discovered after screening chemical libraries. A-769662 has been found to increase AMPK activity through allosteric activation as well as inhibition of dephosphorylation [73]. Furthermore, A-769662 treatment did not alter adenine nucleotide levels nor affect mitochondrial function [74].

Betaine is a metabolite of choline and has previously been shown to be beneficial in alcoholic liver disease. Given its ability to activate AMPK [75], it has also been sought as a treatment for NAFLD. Indeed, an initial pilot study reported beneficial effects of betaine treatment on various parameters of liver function including reduced steatosis and fibrosis.

$\alpha$-Lipoic acid has been shown increase glucose uptake by skeletal muscle and also to activate AMPK in resulting in increased fatty acid oxidation in skeletal muscle [76]. Additionally, part of $\alpha$-Lipoic acid's beneficial effects may be due to activation of PPAR $\alpha$ and FGF21 [77].

AMPK is also modulated by hormones and cytokines such as resistin, leptin, adiponectin, and ghrelin
[53]. The ability of adiponectin to lower hepatic glucose production has been attributed to activation of AMPK [78], as adiponectin was unable to regulate hepatic glucose production in AMPK $\alpha 2$ mice [79]. Adiponectin has been shown to decrease hepatic triglyceride content and thereby restore insulin sensitivity and decrease hepatic fat deposition [80].

\section{Bile-acid receptor and TGR5}

Bile acids (BAs) are critical regulators of hepatic lipid and glucose metabolism and signal through two major receptor pathways: FXR (as discussed above) and TGR5, the G protein-coupled bile acid receptor (GPBAR1). Both FXR and TGR5 demonstrate pleiotropic functions including immune modulation. The activation of BA receptors modulates hepatic monocyte activity and improves non-alcoholic fatty liver disease. McMahanet al [81] reported that obese $\mathrm{db} / \mathrm{db}$ mice were treated with a dual FXR/TGR5 agonist (INT-767) for 6 weeks and the histologic features of NASH in these mice were significantly improved. Furthermore, treatment increased the proportion of intrahepatic monocytes with the anti-inflammatory Ly6Clow phenotype and increased intrahepatic expression of genes expressed by alternatively activated macrophages. In vitro treatment of monocytes with INT-767 led to decreased Ly6C expression and increased IL-10 production through a cAMP-dependent pathway. This indicates that FXR/TGR5 activation may be an important therapeutic target in NAFLD by coordinating the immune phenotype of both monocytes and macrophages.

\section{Nrf2}

Nuclear erythroid 2-related factor 2 (Nrf2) is an oxidative stress-mediated transcription factor with a variety of downstream targets aimed at cytoprotection. Nrf2 has recently been implicated as a new therapeutic target for the treatment of liver disease. Lipid peroxidation has been shown to impair nucleotide and protein synthesis, thereby inducing apoptosis, inflammation, and liver fibrosis. It has been proposed that Nrf2 plays a role in NASH because genetic deletion of Nrf2 in mice results in rapid onset and progression of the disease. There are several stages of disease pathogenesis on which activation of Nrf2 may exert a potential therapeutic effect. The first is in the initial stages of the disease, when lipids are accumulating within the hepatocytes. Nrf2 activation with CDDO-Im has been shown to effectively prevent hepatic lipid accumulation in wildtype mice but not in Nrf2- deficient mice, an effect that may be mediated by a decrease in expression of enzymes required for fatty acid synthesis. Nrf2 may also protect against NAFLD by decreasing inflammation. Several chemotherapeutic agents have been shown in a variety of cell culture and rodent systems to induce Nrf2 and cause simultaneous repression of nuclear factor- $\mathrm{kB}(\mathrm{NF}-\mathrm{\kappa} B$ ), 
an important cell-signaling molecule for the inflammatory response. Additionally, pharmacological activation of Nrf2 results in increased gene expression of Heme oxygenase 1 (Ho-1) and $\mathrm{NAD}(\mathrm{P}) \mathrm{H}$ quinone oxidoreductase 1 (Nqo1), which in turn have inhibitory effects on inflammation [82]. Ho- 1 has been shown in mice to prevent phosphorylation of NF- $\mathrm{kB}$ by its endogenous substrate tumor necrosis factor- $\alpha, 61$ indicating that it has inhibitory effects upstream on the initiation of the inflammatory response. Under normal conditions, lipopolysaccharide(LPS) can be used to induce the expression of tumor necrosis factor- $\alpha$ and cause inflammation through activation of NF- $\kappa B$, however, Nqo1 and Ho-1 overexpression, such as occurs with Nrf2 activation, has been shown in human monocytes to prevent the LPS mediated induction of tumor necrosis factor$\alpha$ expression, thereby preventing inflammation. Another mechanism for the potential treatment of NAFLD and $\mathrm{NASH}$ is through activation of superoxide dismutase and catalase, antioxidant enzymes with decreased activity in this disease state. The pharmaceutical agent and Nrf2 activator Protandim has been shown in human trials to increase erythrocyte superoxide dismutase and catalase activity by 30 and 54\%, respectively. Finally, it is possible that activation of $\mathrm{Nrf} 2$ could play an inhibitory role on transforming growth factor- $\beta$ (TGF- $\beta$ ), a profibrotic signaling factor in plasma, and therefore may inhibit the progression of fibrosis in NASH. A recent study demonstrated that sulforaphane attenuates hepatic fibrosis through Nrf2mediated inhibition of TGF $\beta$ signaling in a human hepatic stellate cell line. This effect was ultimately due to the suppression of hepatic stellate cell activation and fibrogenic gene expression, indicating that activation of Nrf2 has an antifibrotic effect in liver. These findings support a role for Nrf2 signaling in various steps of the pathogenesis of NAFLD.

\section{Mitochondria}

Given their central role in metabolism and fatty acid homeostasis, much attention has recently been focused on the role of mitochondrial function in NAFLD and NASH. However, the exact role of mitochondrial function remains far from clear, and several studies have yielded conflicting results. While a detailed over of all of the studies on the topic is beyond the scope of this review and has been reviewed in detail elsewhere [83-85], we will present a brief overview here. In healthy individuals, lipid uptake and synthesis is relatively well balanced with fatty acid oxidation and lipid export as VLDL being matched by lipid synthesis and lipid uptake [86]. However, insulin resistance and hyperglycemia lead both to increased lipolysis in white adipose tissue which increases the serum fatty acid concentration as well as increased lipid synthesis and uptake by the liver resulting in fat accumulation [87]. Liver mitochondria exhibit the ability to adapt to these changing metabolic conditions and the majority of studies indicate that in various states of disease progression, mitochondria are able to increase fatty acid oxidation in an attempt to counteract liver fat accumulation [88-90]. Various mechanisms underlie this increase in fatty acid oxidation including higher levels of leptin [91], FGF21 [92,93], and IL-6 [94]. PPAR $\alpha$ activation has also been demonstrated $[95,96]$ and can upregulate several genes involved in fatty acid oxidation namely CPT1 [97]. While fatty acid oxidation is able to be upregulated, most of the available evidence has shown that ATP levels are lower in patients with NASH as well as in animal models [98] even though a number of studies have demonstrated that overall oxygen consumption is either increased [99] or unchanged [100]. These seemingly paradoxical results may be due to increased expression of UCP2 in NAFLD resulting in mitochondrial uncoupling [101]. This may well represent a potentially adaptive mechanism to limit reactive oxygen species production which would be presumably induced by the influx of reducing equivalents from increased fatty acid oxidation [102]. However, this mechanism is seemingly insufficient to protect mitochondria from free radical mediated damage as impaired enzymatic activity of various complexes of the electron transport chain has been repeatedly demonstrated $[103,104]$. Several electron transport chain complexes have been shown to be susceptible to inhibition by ROS. ROS can also led to lipid peroxidation which can induce dysfunction of complex IV and the adenine nucleotide translocator as well as altering mitochondrial DNA [105]. Indeed, as increased fatty acid oxidation results in more ROS production and damage to the electron transport chain, a further mismatch between the two likely occurs presumably resulting in progressively more electrons being lost as free radicals [102].

Therapeutic strategies to target mitochondria as treatments for NAFLD and NASH are still in the very early stages. Approaches to further increase fatty acid oxidation have been proposed in order to decrease the accumulation of fat in the liver [106], however this approach may further exacerbate the imbalance between fatty acid oxidation and the electron transport chain further increasing ROS production. To this point, inhibition of CPT-I, which catalyzes the rate-limiting step of fatty acid oxidation, has been shown to prevent high fat diet induced insulin resistance, in part due to a reduction in some of the deleterious intermediates generated by incomplete fatty acid oxidation and also due to a shift toward increased glucose oxidation for energy production [107]. Therefore, the development of viable therapeutics targeting mitochondrial pathways will likely be more complicated than anticipated and should take into account not only the effect on the liver but also potential effect on the metabolic pathways of other tissues. Nevertheless, 
some compounds shown to improve mitochondrial function have shown initial promise in treating NAFLD.

Curcumin, which is known to have hepatoprotective effects, was shown to inhibit the progression of hepatic steatosis in a rabbit model by improving mitochondrial respiratory chain function, decreasing oxidative stress, and reducing levels of TNF- $\alpha$ [108]. Similarly, silybin, which has antioxidant, anti-inflammatory, and anti-fibrotic activity, has been shown to protect against oxidative stress, preserve mitochondrial function, and limit the progression of NASH in an animal model [109]. Given their already widespread use and minimal side effect profile, other antioxidants such as vitamin $\mathrm{E}$, vitamin $\mathrm{C}$, and $\mathrm{N}$ acetylcysteine have been studied in models of NASH with favorable outcomes [110]. While extensive clinical data demonstrating the beneficial effect of antioxidants in $\mathrm{NASH}$ remain to be generated, early studies have shown promise. For instance, a silybin-vitamin E compound has been shown to improve various parameters of liver function in patients with NAFLD.

\section{Cannabinoid receptors}

Cannabinoids exert their effects through two different cannabinoid receptors: CB1 and CB2, both of which are G-protein coupled. Both of these receptors have been implicated in the development of liver fibrosis secondary to various etiologies. It has been shown that marijuana use may correlate with the progression of liver fibrosis in patients with hepatitis $C$ [111]. However, each receptor seems to have opposing roles in the liver. The $\mathrm{rCB} 2$ recepto has been shown to be up-regulated in the livers of cirrhotic patients and has been shown to ameliorate the progression of fibrosis [112]. In contrast, CB1 receptor activation has been linked to the progression of fibrosis and CB1 antagonists have been shown to inhibit the progression of fibrosis [113]. Indeed, clinical trials with a CB1 receptor antagonist have shown that antagonism of CB1 can result in weight loss and improved metabolic and cardiac parameters in overweight and obese populations [114]. However, given considerable side effects, future compounds developed for the treatment of NASH will likely need to be peripherally targeted [115].

Cannabinoid receptors are have emerged as major targets for the treatment of obesity. New compounds which target cannabinoid receptors have been developed for weight loss and may prove to be efficacious in the management of NAFLD. Experimental and clinical data indicate that peripheral activation of cannabinoid CB1 receptors promotes insulin resistance and liver steatogenesis, two key steps in the pathogenesis of non-alcoholic fatty liver disease. Moreover, activation of CB1 receptorsenhance progression of liver fibrogenesis. These findings provide the rationale for the use of CB1 antagonists in the management of NAFLD or NASH.

\section{Conclusions}

In this review, we have outlined some of the molecular pathways contributing to the pathogenesis of NAFLD. While the details of many of these pathways are still being elucidated, they provide targets for future therapeutic strategies in NAFLD. Still, much work remains to be done in detailing the exact mechanisms contributing to NALD and the coordination and interaction among the various pathways that have already been implicated in disease pathogenesis.

\section{Competing interests}

The authors declare that they have no competing interests.

\section{Authors' contributions}

SQ wrote the manuscript; YYH provided all the reference and also wrote the manuscript; AMG was involved in editing the manuscript. All authors read and approved the final manuscript.

\section{Acknowledgements}

This review was supported by Shanghai CDC Grant(SHDC12012303) and CSE grant 13020260411 .

\section{Author details}

'Department of Endocrinology and Metabolism, Shanghai 10th People's Hospital, School of Medicine, Tongji University, Shanghai 200072, China. 2Department of Endocrinology and Metabolism, Nanjing Medical University, Nanjing 210029, Jiangsu Province, China. ${ }^{3}$ Department of Neurology and Neuroscience, Weill Cornell Medical College, New York, NY 10065, USA.

Received: 25 September 2013 Accepted: 7 November 2013

Published: 9 November 2013

\section{References}

1. Zhou J, et al: Hepatic fatty acid transporter $\mathrm{Cd} 36$ is a common target of LXR, PXR, and PPARgamma in promoting steatosis. Gastroenterology 2008, 134(2):556-567.

2. Blumberg $B$, et al: SXR, a novel steroid and xenobiotic-sensing nuclear receptor. Genes Dev 1998, 12(20):3195-3205.

3. Timsit YE, Negishi M: CAR and PXR: the xenobiotic-sensing receptors Steroids 2007, 72(3):231-246.

4. Zhou J, et al: A novel pregnane $\mathrm{X}$ receptor-mediated and sterol regulatory element-binding protein-independent lipogenic pathway. J Biol Chem 2006, 281(21):15013-15020.

5. Moreau A, et al: A novel pregnane $X$ receptor and S14-mediated lipogenic pathway in human hepatocyte. Hepatology 2009, 49(6):2068-2079.

6. Wolfrum C, Asilmaz E, Luca E, Friedman JM, Stoffel M: Foxa2 regulates lipid metabolism and ketogenesis in the liver during fasting and in diabetes. Nature 2004, 432(7020):1027-1032.

7. Nakamura K, Moore R, Negishi M, Sueyoshi T: Nuclear pregnane X receptor cross-talk with FoxA2 to mediate drug-induced regulation of lipid metabolism in fasting mouse liver. J Biol Chem 2007, 282(13):9768-9776.

8. Valenti $\mathrm{L}$, et al: Increased expression and activity of the transcription factor FOXO1 in nonalcoholic steatohepatitis. Diabetes 2008, 57(5):1355-1362.

9. Kodama S, Koike C, Negishi M, Yamamoto Y: Nuclear receptors CAR and PXR cross talk with FOXO1 to regulate genes that encode drug-metabolizing and gluconeogenic enzymes. Mol Cell Biol 2004, 24(18):7931-7940.

10. Parks DJ, et al: Bile acids: natural ligands for an orphan nuclear receptor. Science 1999, 284(5418):1365-1368.

11. Downes $M$, et al: A chemical, genetic, and structural analysis of the nuclear bile acid receptor FXR. Mol Cell 2003, 11(4):1079-1092.

12. Thomas C, Pellicciari R, Pruzanski M, Auwerx J, Schoonjans K: Targeting bile-acid signalling for metabolic diseases. Nat Rev Drug Discov 2008, 7(8):678-693.

13. Musso G, Gambino R, Cassader M: Emerging molecular targets for the treatment of nonalcoholic fatty liver disease. Annu Rev Med 2010, 61:375-392. 
14. Sinal CJ, et al: Targeted disruption of the nuclear receptor FXR/BAR impairs bile acid and lipid homeostasis. Cell 2000, 102(6):731-744.

15. Kong B, Luyendyk JP, Tawfik O, Guo GL: Farnesoid X receptor deficiency induces nonalcoholic steatohepatitis in low-density lipoprotein receptor-knockout mice fed a high-fat diet. J Pharmacol Exp Ther 2009, 328(1):116-122

16. Zhang Y, Castellani LW, Sinal CJ, Gonzalez FJ, Edwards PA: Peroxisome proliferator-activated receptor-gamma coactivator 1alpha (PGC-1alpha) regulates triglyceride metabolism by activation of the nuclear receptor FXR. Genes Dev 2004, 18(2):157-169.

17. Claudel $T$, et al: Farnesoid $X$ receptor agonists suppress hepatic apolipoprotein CIII expression. Gastroenterology 2003, 125(2):544-555.

18. Yamagata $\mathrm{K}$, et al: Bile acids regulate gluconeogenic gene expression via small heterodimer partner-mediated repression of hepatocyte nuclear factor 4 and Foxo1. J Biol Chem 2004, 279(22):23158-23165.

19. Ma K, Saha PK, Chan L, Moore DD: Farnesoid X receptor is essential for normal glucose homeostasis. J Clin Invest 2006, 116(4):1102-1109.

20. Inagaki T, et al: Regulation of antibacterial defense in the small intestine by the nuclear bile acid receptor. Proc Natl Acad Sci USA 2006, 103(10):3920-3925

21. Chakravarthy MV, et al: Identification of a physiologically relevant endogenous ligand for PPARalpha in liver. Cell 2009, 138(3):476-488.

22. Okamura M, Inagaki T, Tanaka T, Sakai J: Role of histone methylation and demethylation in adipogenesis and obesity. Organogenesis 2010, 6(1):24-32.

23. Nissen SE, Wolski K: Rosiglitazone revisited: an updated meta-analysis of risk for myocardial infarction and cardiovascular mortality. Arch Intern Med 2010, 170(14):1191-1201.

24. Dongiovanni P, Valenti L: Peroxisome proliferator-activated receptor genetic polymorphisms and nonalcoholic Fatty liver disease: any role in disease susceptibility? PPAR Research 2013, 2013:452061

25. Sahebkar A: Does PPARgamma2 gene Pro12Ala polymorphism affect nonalcoholic fatty liver disease risk? Evidence from a meta-analysis. DNA Cell Biol 2013, 32(4):188-198.

26. Wang J, et al: Association between the Pro12Ala polymorphism of PPARgamma gene and the non-alcoholic fatty liver disease: a meta-analysis. Gene 2013, 528(2):328-334.

27. Lazarow PB, Fujiki Y: Biogenesis of peroxisomes. Annu Rev Cell Biol 1985, 1:489-530.

28. Wanders RJ, Tager JM: Lipid metabolism in peroxisomes in relation to human disease. Mol Aspects Med 1998, 19(2):69-154.

29. Wanders RJ, Waterham HR: Biochemistry of mammalian peroxisomes revisited. Annu Rev Biochem 2006, 75:295-332

30. Abe I, Okumoto K, Tamura S, Fujiki Y: Clofibrate-inducible, 28-kDa peroxisomal integral membrane protein is encoded by PEX11. FEBS Lett 1998, 431(3):468-472

31. Delille HK, et al: Pex11pbeta-mediated growth and division of mammalian peroxisomes follows a maturation pathway. J Cell Sci 2010, 123(Pt 16):2750-2762.

32. Koch J, et al: PEX11 family members are membrane elongation factors that coordinate peroxisome proliferation and maintenance. J Cell Sci 2010, 123(Pt 19):3389-3400.

33. Marshall PA, et al: Pmp27 promotes peroxisomal proliferation. J Cell Biol 1995, 129(2):345-355.

34. Schrader $\mathrm{M}$, et al: Expression of PEX11beta mediates peroxisome proliferation in the absence of extracellular stimuli. J Biol Chem 1998, 273(45):29607-29614.

35. Li X, et al: PEX11alpha is required for peroxisome proliferation in response to 4-phenylbutyrate but is dispensable for peroxisome proliferator-activated receptor alpha-mediated peroxisome proliferation Mol Cell Biol 2002, 22(23):8226-8240.

36. Weng $H$, et al: Pex11alpha deficiency impairs peroxisome elongation and division and contributes to nonalcoholic fatty liver in mice. Am J Physiol Endocrinol Metab 2013, 304(2):E187-E196.

37. Ma W, Sung HJ, Park JY, Matoba S, Hwang PM: A pivotal role for p53: balancing aerobic respiration and glycolysis. J Bioenerg Biomembr 2007, 39(3):243-246

38. Yahagi $N$, et al: p53 involvement in the pathogenesis of fatty liver disease. J Biol Chem 2004, 279(20):20571-20575.

39. Farrell GC, et al: Apoptosis in experimental NASH is associated with p53 activation and TRAIL receptor expression. J Gastroenterol Hepatol 2009, 24(3):443-452
40. Derdak $Z$, et al: Inhibition of p53 attenuates steatosis and liver injury in a mouse model of non-alcoholic fatty liver disease. J Hepatol 2013, 58(4):785-791.

41. Castro RE, et al: miR-34a/SIRT1/p53 is suppressed by ursodeoxycholic acid in the rat liver and activated by disease severity in human non-alcoholic fatty liver disease. J Hepatol 2013, 58(1):119-125.

42. Pommier AJ, et al: Liver $x$ receptors protect from development of prostatic intra-epithelial neoplasia in mice. PLoS Genet 2013, 9(5):e1003483

43. Zelcer N, Hong C, Boyadjian R, Tontonoz P: LXR Regulates Cholesterol Uptake Through Idol-Dependent Ubiquitination of the LDL Receptor. Science 2009, 325(5936):100-104.

44. Suckling K: Selective thyromimetics for atherosclerosis and dyslipidaemia: another old target making progress. Expert Opin Investig Drugs 2008, 17(5):615-618

45. Berkenstam A, et al: The thyroid hormone mimetic compound KB2115 lowers plasma LDL cholesterol and stimulates bile acid synthesis without cardiac effects in humans. Proc Natl Acad Sci USA 2008, 105(2):663-667.

46. Cable $\mathrm{EE}$, et al: Reduction of hepatic steatosis in rats and mice after treatment with a liver-targeted thyroid hormone receptor agonist. Hepatology 2009, 49(2):407-417.

47. Grant N: The role of triiodothyronine-induced substrate cycles in the hepatic response to overnutrition: thyroid hormone as an antioxidant. Med Hypotheses 2007, 68(3):641-649.

48. Hardie DG: AMP-activated protein kinase: an energy sensor that regulates all aspects of cell function. Genes Dev 2011, 25(18):1895-1908.

49. Barnes K, et al: Activation of GLUT1 by metabolic and osmotic stress: potential involvement of AMP-activated protein kinase (AMPK). J Cell SCi 2002, 115(Pt 11):2433-2442.

50. Marsin AS, et al: Phosphorylation and activation of heart PFK-2 by AMPK has a role in the stimulation of glycolysis during ischaemia. Current Biology: CB 2000, 10(20):1247-1255

51. Mihaylova MM, et al: Class lla histone deacetylases are hormone-activated regulators of FOXO and mammalian glucose homeostasis. Cell 2011, 145(4):607-621.

52. Jorgensen SB, et al: The alpha2-5'AMP-activated protein kinase is a site 2 glycogen synthase kinase in skeletal muscle and is responsive to glucose loading. Diabetes 2004, 53(12):3074-3081.

53. Koo SH, et al: The CREB coactivator TORC2 is a key regulator of fasting glucose metabolism. Nature 2005, 437(7062):1109-1111.

54. Foretz $M$, et al: Short-term overexpression of a constitutively active form of AMP-activated protein kinase in the liver leads to mild hypoglycemia and fatty liver. Diabetes 2005, 54(5):1331-1339.

55. Jager S, Handschin C, St-Pierre J, Spiegelman BM: AMP-activated protein kinase (AMPK) action in skeletal muscle via direct phosphorylation of PGC-1alpha. Proc Natl Acad Sci USA 2007, 104(29):12017-12022.

56. Narkar VA, et al: AMPK and PPARdelta agonists are exercise mimetics. Cell 2008, 134(3):405-415.

57. Jorgensen SB, et al: Effects of alpha-AMPK knockout on exercise-induced gene activation in mouse skeletal muscle. FASEB J 2005, 19(9):1146-1148.

58. Zhang $W$, et al: Thiazolidinediones improve hepatic fibrosis in rats with non-alcoholic steatohepatitis by activating the adenosine monophosphateactivated protein kinase signalling pathway. Clin Exp Pharmacol Physiol 2012, 39(12):1026-1033

59. Zhou G, et al: Role of AMP-activated protein kinase in mechanism of metformin action. J Clin Invest 2001, 108(8):1167-1174.

60. Zang $M$, et al: AMP-activated protein kinase is required for the lipidlowering effect of metformin in insulin-resistant human HepG2 cells. J Biol Chem 2004, 279(46):47898-47905.

61. Garcia-Ruiz I, Solis-Munoz P, Fernandez-Moreira D, Munoz-Yague T, SolisHerruzo JA: Pioglitazone leads to an inactivation and disassembly of complex I of the mitochondrial respiratory chain. BMC Biol 2013, 11:88

62. Divakaruni AS, et al: Thiazolidinediones are acute, specific inhibitors of the mitochondrial pyruvate carrier. Proc Natl Acad Sci USA 2013, 110(14):5422-5427.

63. Baur JA, et al: Resveratrol improves health and survival of mice on a high-calorie diet. Nature 2006, 444(7117):337-342.

64. Hwang JT, et al: Apoptotic effect of EGCG in HT-29 colon cancer cells via AMPK signal pathway. Cancer Lett 2007, 247(1):115-121.

65. Hou X, et al: SIRT1 regulates hepatocyte lipid metabolism through activating AMP-activated protein kinase. J Biol Chem 2008, 283(29):20015-20026. 
66. Collins QF, et al: Epigallocatechin-3-gallate (EGCG), a green tea polyphenol, suppresses hepatic gluconeogenesis through 5'-AMP-activated protein kinase. J Biol Chem 2007, 282(41):30143-30149.

67. Gledhill JR, Montgomery MG, Leslie AG, Walker JE: Mechanism of inhibition of bovine F1-ATPase by resveratrol and related polyphenols. Proc Natl Acad Sci USA 2007, 104(34):13632-13637.

68. Valenti $D$, et al: Negative modulation of mitochondrial oxidative phosphorylation by epigallocatechin-3 gallate leads to growth arrest and apoptosis in human malignant pleural mesothelioma cells. Biochimica et Piophysica Acta 2013, 1832(12):2085-2096.

69. Bergeron $\mathrm{R}$, et al: Effect of 5-aminoimidazole-4-carboxamide-1-beta-Dribofuranoside infusion on in vivo glucose and lipid metabolism in lean and obese Zucker rats. Diabetes 2001, 50(5):1076-1082.

70. Taylor EB, et al: Discovery of TBC1D1 as an insulin-, AICAR-, and contraction-stimulated signaling nexus in mouse skeletal muscle. J Biol Chem 2008, 283(15):9787-9796.

71. Mukhtar MH, et al: Inhibition of glucokinase translocation by AMPactivated protein kinase is associated with phosphorylation of both GKRP and 6-phosphofructo-2-kinase/fructose-2,6-bisphosphatase. Am J Physiol Regul Integr Comp Physiol 2008, 294(3):R766-R774.

72. Guigas $B$, et al: AMP-activated protein kinase-independent inhibition of hepatic mitochondrial oxidative phosphorylation by AICA riboside. Biochem J 2007, 404(3):499-507.

73. Sanders MJ, Grondin PO, Hegarty BD, Snowden MA, Carling D: Investigating the mechanism for AMP activation of the AMP-activated protein kinase cascade. Biochem J 2007, 403(1):139-148.

74. Guigas B, et al: Beyond AICA riboside: in search of new specific AMPactivated protein kinase activators. IUBMB life 2009, 61(1):18-26.

75. Song $Z$, et al: Involvement of AMP-activated protein kinase in beneficial effects of betaine on high-sucrose diet-induced hepatic steatosis. Am J Physiol Gastrointest Liver Physiol 2007, 293(4):G894-G902.

76. Lee WJ, et al: Alpha-lipoic acid increases insulin sensitivity by activating AMPK in skeletal muscle. Biochem Biophys Res Commun 2005, 332(3):885-891.

77. Yi X, Pashaj A, Xia M, Moreau R: Reversal of obesity-induced hypertriglyceridemia by (R)-alpha-lipoic acid in ZDF (fa/fa) rats. Biochemical and biophysical research communications. 2013. http://www.ncbi.nlm.nih.gov/pubmed/?term= Reversal+of+obesity-induced+hypertriglyceridemia+by+(R)-alpha-lipoic+acid+ in+ZDF+(fa\%2Ffa)+rats.

78. Yamauchi T, et al: Targeted disruption of AdipoR1 and AdipoR2 causes abrogation of adiponectin binding and metabolic actions. Nat Med 2007, 13(3):332-339.

79. Andreelli $F$, et al: Liver adenosine monophosphate-activated kinasealpha2 catalytic subunit is a key target for the control of hepatic glucose production by adiponectin and leptin but not insulin. Endocrinology 2006, 147(5):2432-2441.

80. $\mathrm{Xu}$ A, et al: The fat-derived hormone adiponectin alleviates alcoholic and nonalcoholic fatty liver diseases in mice. J Clin Invest 2003, 112(1):91-100.

81. McMahan $\mathrm{RH}$, et al: Bile acid receptor activation modulates hepatic monocyte activity and improves nonalcoholic fatty liver disease. J Biol Chem 2013, 288(17):11761-11770.

82. Bataille AM, Manautou JE: Nrf2: A Potential Target for New Therapeutics in Liver Disease. Clin Pharmacol Ther 2012, 92(3):340-348.

83. Begriche K, Massart J, Robin MA, Bonnet F, Fromenty B: Mitochondrial adaptations and dysfunctions in nonalcoholic fatty liver disease. Hepatology 2013, 58(4):1497-1507.

84. Garcia-Ruiz C, Baulies A, Mari M, Garcia-Roves PM, Fernandez-Checa JC: Mitochondrial dysfunction in non-alcoholic fatty liver disease and insulin resistance: Cause or consequence? Free Radic Res 2013, 47(11):854-868

85. Koliaki C, Roden M: Hepatic energy metabolism in human diabetes mellitus, obesity and non-alcoholic fatty liver disease. / Cell Endocrinol 2013, 379(1-2):35-42.

86. Fabbrini E, Sullivan S, Klein S: Obesity and nonalcoholic fatty liver disease: biochemical, metabolic, and clinical implications. Hepatology 2010, 51(2):679-689.

87. Tamura S, Shimomura I: Contribution of adipose tissue and de novo lipogenesis to nonalcoholic fatty liver disease. J Clin Invest 2005, 115(5):1139-1142.

88. Crescenzo R, et al: Increased hepatic de novo lipogenesis and mitochondrial efficiency in a model of obesity induced by diets rich in fructose. Eur J Nutr 2013, 52(2):537-545.
89. Ciapaite J, et al: Differential effects of short- and long-term high-fat diet feeding on hepatic fatty acid metabolism in rats. Biochim Biophys Acta 2011, 1811(7-8):441-451

90. Dasarathy $S$, et al: Elevated hepatic fatty acid oxidation, high plasma fibroblast growth factor 21, and fasting bile acids in nonalcoholic steatohepatitis. Eur J Gastroenterol Hepatol 2011, 23(5):382-388.

91. Myers MG Jr, Leibel RL, Seeley RJ, Schwartz MW: Obesity and leptin resistance: distinguishing cause from effect. Trends Endocrinol Metab 2010, 21(11):643-651.

92. Dushay J, et al: Increased fibroblast growth factor 21 in obesity and nonalcoholic fatty liver disease. Gastroenterology 2010, 139(2):456-463.

93. Li H, et al: Fibroblast growth factor 21 levels are increased in nonalcoholic fatty liver disease patients and are correlated with hepatic triglyceride. J Hepatol 2010, 53(5):934-940.

94. Matthews VB, et al: Interleukin-6-deficient mice develop hepatic inflammation and systemic insulin resistance. Diabetologia 2010, 53(11):2431-2441.

95. Takamura $T$, et al: Obesity upregulates genes involved in oxidative phosphorylation in livers of diabetic patients. Obesity 2008, 16(12):2601-2609.

96. Alves TC, et al: Regulation of hepatic fat and glucose oxidation in rats with lipid-induced hepatic insulin resistance. Hepatology 2011, 53(4):1175-1181.

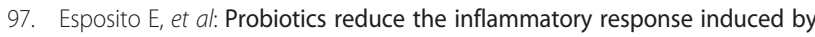
a high-fat diet in the liver of young rats. J Nutr 2009, 139(5):905-911.

98. Serviddio $\mathrm{G}$, et al: Alterations of hepatic ATP homeostasis and respiratory chain during development of non-alcoholic steatohepatitis in a rodent model. Eur J Clin Invest 2008, 38(4):245-252

99. Lazarin Mde $\mathrm{O}$, et al: Liver mitochondrial function and redox status in an experimental model of non-alcoholic fatty liver disease induced by monosodium L-glutamate in rats. Exp Mol Pathol 2011, 91(3):687-694

100. Flamment $M$, et al: Regulation of hepatic mitochondrial metabolism in response to a high fat diet: a longitudinal study in rats. $J$ Physiol Biochem 2012, 68(3):335-344.

101. Jiang $Y$, Zhang $H$, Dong LY, Wang D, An W: Increased hepatic UCP2 expression in rats with nonalcoholic steatohepatitis is associated with upregulation of $\mathrm{Sp} 1$ binding to its motif within the proximal promoter region. J Cell Biochem 2008, 105(1):277-289.

102. Begriche K, Massart J, Robin MA, Borgne-Sanchez A, Fromenty B: Druginduced toxicity on mitochondria and lipid metabolism: mechanistic diversity and deleterious consequences for the liver. J Hepatol 2011, 54(4):773-794.

103. Mingorance $C$, et al: Propionyl-L-carnitine corrects metabolic and cardiovascular alterations in diet-induced obese mice and improves liver respiratory chain activity. PloS one 2012, 7(3):e34268.

104. Finocchietto PV, et al: Defective leptin-AMP-dependent kinase pathway induces nitric oxide release and contributes to mitochondrial dysfunction and obesity in ob/ob mice. Antioxid Redox Signal 2011, 15(9):2395-2406

105. Pessayre D: Role of mitochondria in non-alcoholic fatty liver disease. J Gastroenterol Hepatol 2007, 22(Suppl 1):S20-S27.

106. Mollica MP, et al: 3,5-diiodo-l-thyronine, by modulating mitochondrial functions, reverses hepatic fat accumulation in rats fed a high-fat diet. J Hepatol 2009, 51(2):363-370.

107. Hancock CR, et al: High-fat diets cause insulin resistance despite an increase in muscle mitochondria. Proc Natl Acad Sci USA 2008, 105(22):7815-7820.

108. Ramirez-Tortosa MC, et al: Curcumin ameliorates rabbits's steatohepatitis via respiratory chain, oxidative stress, and TNF-alpha. Free Radic Biol Med 2009, 47(7):924-931.

109. Serviddio $G$, et al: A silybin-phospholipid complex prevents mitochondrial dysfunction in a rodent model of nonalcoholic steatohepatitis. J Pharmacol Exp Ther 2010, 332(3):922-932.

110. Samuhasaneeto S, Thong-Ngam D, Kulaputana O, Patumraj S, Klaikeaw N: Effects of $\mathrm{N}$-acetylcysteine on oxidative stress in rats with non-alcoholic steatohepatitis. J Med Assoc Thai 2007, 90(4):788-797.

111. Hezode C, et al: Daily cannabis smoking as a risk factor for progression of fibrosis in chronic hepatitis C. Hepatology 2005, 42(1):63-71.

112. Munoz-Luque J, et al: Regression of fibrosis after chronic stimulation of cannabinoid CB2 receptor in cirrhotic rats. J Pharmacol Exp Ther 2008, 324(2):475-483.

113. Teixeira-Clerc F, et al: CB1 cannabinoid receptor antagonism: a new strategy for the treatment of liver fibrosis. Nat Med 2006, 12(6):671-676. 
114. Van Gaal L, Pi-Sunyer X, Despres JP, McCarthy C, Scheen A: Efficacy and safety of rimonabant for improvement of multiple cardiometabolic risk factors in overweight/obese patients: pooled 1-year data from the Rimonabant in Obesity (RIO) program. Diabetes Care 2008, 31(Suppl 2):S229-S240.

115. Janero DR, Lindsley L, Vemuri VK, Makriyannis A: Cannabinoid $1 \mathrm{G}$ proteincoupled receptor (periphero-)neutral antagonists: emerging therapeutics for treating obesity-driven metabolic disease and reducing cardiovascular risk. Expert Opinion on Drug Discovery 2011, 6(10):995-1025.

doi:10.1186/1476-511X-12-171

Cite this article as: Huang et al:: Nonalcoholic fatty liver disease: molecular pathways and therapeutic strategies. Lipids in Health and Disease 2013 12:171.

\section{Submit your next manuscript to BioMed Central and take full advantage of:}

- Convenient online submission

- Thorough peer review

- No space constraints or color figure charges

- Immediate publication on acceptance

- Inclusion in PubMed, CAS, Scopus and Google Scholar

- Research which is freely available for redistribution 